\title{
MOST Photometry of the roAp star HD 134214
}

\author{
C. Cameron ${ }^{1}$, J.M. Matthews ${ }^{1}$, J.F. Rowe ${ }^{1}$, R. Kuschnig ${ }^{1}$, D.B. Guenther ${ }^{2}$, \\ A.F.J. Moffat ${ }^{3}$, S.M. Rucinski ${ }^{4}$, D. Sasselov ${ }^{5}$, G.A.H., Walker ${ }^{6}$, W.W. Weiss ${ }^{7}$ \\ ${ }^{1}$ Dept. of Physics and Astronomy, UBC, 6224 Agricultural Road, \\ Vancouver, BC, V6T 1Z1, Canada \\ ${ }^{2}$ Dept. of Astronomy and Physics, Saint Mary's University, \\ Halifax, NS, B3H 3C3, Canada \\ ${ }^{3}$ Dépt. de physique, Univ. de Montréal C.P. 6128, Succ. Centre-Ville, \\ Montréal, QC H3C 3J7, Canada; and Obs. du Mont Mégantic \\ ${ }^{4}$ Dept. of Astronomy \& Astrophysics, David Dunlap Obs., Univ. Toronto P.O. Box \\ 360, Richmond Hill, ON L4C 4Y6, Canada \\ ${ }^{5}$ Harvard-Smithsonian Center for Astrophysics, 60 Garden Street, \\ Cambridge, MA 02138, USA \\ ${ }^{6} 1234$ Hewlett Place, Victoria, BC V8S 4P7, Canada \\ ${ }^{7}$ Institut für Astronomie, Universität Wien Türkenschanzstrasse 17, \\ A-1180 Wien, Austria
}

\begin{abstract}
We present $10.27 \mathrm{hrs}$ of photometry of the roAp star HD 134214 obtained by the $M O S T^{1}$ satellite. The star is shown to be monoperiodic and oscillating at a frequency of $2948.97 \pm 0.55 \mu \mathrm{Hz}$. This is consistent with earlier ground based photometric campaigns (e.g. Kreidl et al. 1994). We do not detect any of the additional frequencies identified in the recent spectroscopic study by Kurtz et al. (2006) down to an amplitude limit of $0.36 \mathrm{mmag}$ ( $2 \sigma$ significance limit).
\end{abstract}

\section{Introduction}

The rapidly oscillating Ap stars (roAp) represent a unique subset of the chemically peculiar stars of the upper main sequence. In general, the Ap stars have

\footnotetext{
${ }^{1}$ MOST (Microvariability and Oscillations of STars) is a Canadian Space Agency mission, operated jointly by Dynacon, Inc., and the Universities of Toronto and British Columbia, with assistance from the University of Vienna.
} 
globally organised magnetic fields of strengths of order kiloGauss, and spectral anomalies that are interpreted as vertical and horizontal chemical inhomogeneities in the stellar atmosphere. The roAp stars (among the coolest members of the Ap class) exhibit rapid oscillations in photometry and spectroscopy. These variations (first observed by Kurtz (1982)) have periods from about 5 to 20 minutes and low amplitudes $(B \lesssim 10 \mathrm{mmag}$ ). They are consistent with acoustic ( $\mathrm{p}$-mode) pulsations of low degree and high radial overtone. A thorough review of the roAp stars is provided by Kurtz \& Martinez (2000).

HD 134214 is in many ways a typical Ap star. It has an effective temperature of $\sim 7500 \mathrm{~K}$, is a moderately slow rotator with a $v \sin i \approx 2.0 \mathrm{~km} / \mathrm{s}$, and has a strong magnetic field with $B_{r} \approx-2.9 \mathrm{kG}$ (parameters are derived in Shavrina et al. 2004). However, among the roAp stars, HD 134214 stands alone as the star with the shortest known period ( $\sim 5.6$ minutes). This high frequency oscillation is well above the estimated isothermal acoustic cutoff for a typical A-star model (see, e.g., Audard et al. 1998). There is also evidence that this oscillation frequency is variable at the level of a few tenths of $\mu \mathrm{Hz}$ over a time scale of approximately one year, even though the amplitude remained stable over many years (see Kreidl et al. 1994). Kurtz (1995) discuss the frequency variability observed in this and other roAp stars.

Recently, Kurtz et al. (2006) have shown that in some roAp stars the spectroscopic variability is dramatically different from that observed photometrically. Although HD 134214 is observed to be monoperiodic in photometry over many years (Kreidl et al. 1994), Kurtz et al. present evidence for up to 6 periodicities in their (admittedly short) spectroscopic time-series of this star.

In this paper we present MOST (Microvariability \& Oscillations of STars) spacebased photometry of HD 134214 and compare the observed oscillations to the results of Kurtz et al. (2006).

\section{Photometry and Frequency Analysis}

MOST is a Canadian microsatellite that was launched into a $820-\mathrm{km}$ Sunsynchronous polar orbit in June 2003. Its primary science objective is to obtain nearly continuous, ultra-precise photometric measurements of stars for the purposes of asteroseismology. The instrument is a 15-cm Rumak-Maksutov telescope that illuminates a CCD photometer through a custom broadband filter (350-700 nm). Detailed technical information on the instrument is contained in Walker et al. (2003). The first science results for the mission were published by Matthews et al. (2004).

The roAp star HD 134214 was observed by MOST as a trial Direct Imaging target on 1 May 2006. Direct Imaging is the observing mode where a defocused star image is projected onto an open area of the Science CCD (see 
Rowe et al. 2006a and references therein for more details on different observing modes of MOST). The star was in one of two target fields observed during each MOST satellite orbit (period $=101.413 \mathrm{~min}$ ), so data are collected with a duty cycle of only $\sim 47 \%$. Exposures are $1.5 \mathrm{sec}$ long, obtained every 10 seconds. There were a total of 505 measurements collected during 10.27 hrs.

Photometric reductions for Direct Imaging targets are described by Rowe et al. (2006b). In addition to these procedures, the data are detrended using a running mean of approximately 15 mins to reduce any remaining low-frequency contributions to stray light from scattered Earthshine which is modulated with the MOST satellite orbital period. This detrending does not influence the high-frequency domain of interest for roAp oscillations. The resulting changes in the mean level of the light curve are not large enough to affect the measured amplitude of the oscillation signal, within the uncertainty from the fit (see below).

The reduced photometry is presented in Figure 1. The light curve of HD 134214 , shown in the top panel, has a standard deviation of $\sim 1.8 \mathrm{mmag}$ and a $2 \sigma$ standard error of 0.2 mmag. Data subsets labelled A and B are shown in the lower two panels.

Frequency determinations are made using CAPER; see Walker et al. (2005) and Saio et al. (2006). CAPER is an iterative procedure that identifies periodicities in Fourier space and simultaneously fits a set of sinusoids in the time domain. This method of determining oscillation parameters from timeseries data follows the philosophy of the popular software packages Period98 (Sperl 1998) and Period04 (Lenz \& Breger 2005). Discrete Fourier Transforms (DFTs) are calculated and the fit is subtracted from the data successively, until there is no meaningful change in the fit residuals.

The DFT and spectral window function for the HD 134214 data are shown in Figure 2. There is one periodicity identified at $2948.97 \pm 0.55 \mu \mathrm{Hz}$ with a signal-to-noise ratio of 15.4. The noise in the Fourier domain was estimated as the mean of the entire spectrum $(0.12 \mathrm{mmag})$. We see no evidence for other periodicities at a detection threshold of about $2 \sigma$ (or $3 \times$ the noise; see Kuschnig et al. 1997). The fitted values of amplitude and phase are $1.88 \pm 0.09 \mathrm{mmag}$ and $1.97 \pm 0.39$ radians respectively. The uncertainties in the fit parameters are determined using a bootstrap technique (see below). The dark line in Figure 1 shows the fit of this single sinusoid plotted over the observations.

The uncertainties we calculate for time-series parameters derived from nonlinear least-squares methods depend on the noise of the data (which may be a combination of instrumental and random processes) and on the time sampling. It is also known that fitted phase and frequency parameters are correlated, leading to underestimated uncertainties in these parameters when calculated from 

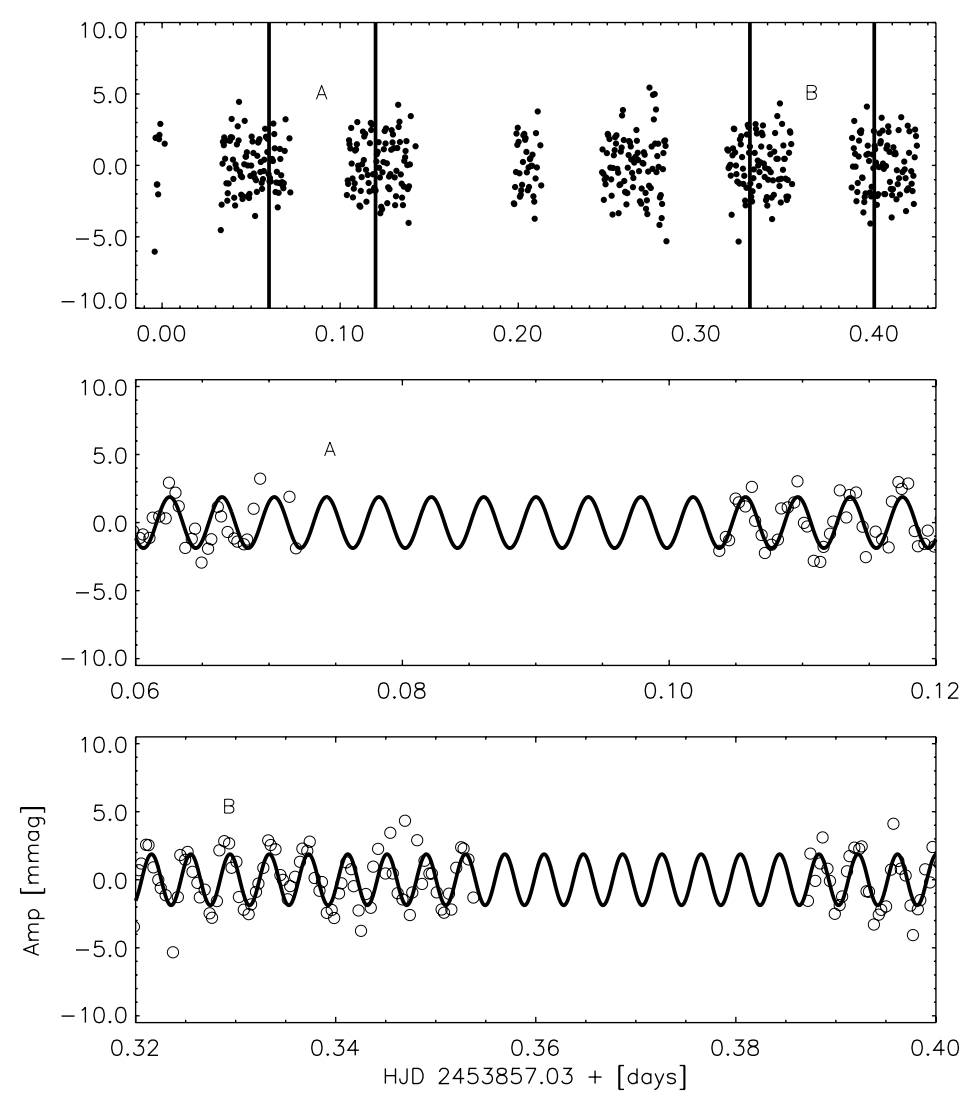

Figure 1: The MOST photometry of HD 134214. The top panel shows the $10.27 \mathrm{hrs}$ of data. Gaps in the light curve are the result of observing this target every second satellite orbit. The zoomed regions; labelled $A$ and $B$, are shown respectively in the lower panels. The open circles are approximately twice the standard error in size ( $\sim 0.20 \mathrm{mmag})$. The solid line represents the fit of a single sinusoid with a frequency of $2948.97 \mu \mathrm{Hz}$.

a covariance matrix (e.g., Montgomery \& O'Donoghue 1999).

The "bootstrap" is an ideal way to assess the uncertainties in fitted parameters for a time-series analysis. The procedure is outlined in Wall \& Jenkins (2003) and has recently been used in a number of MOST applications (e.g., Rowe et al. 2006b and Saio et al. 2006). Clement et al. (1992) also used a bootstrap to estimate the uncertainties in Fourier parameters they derived for 

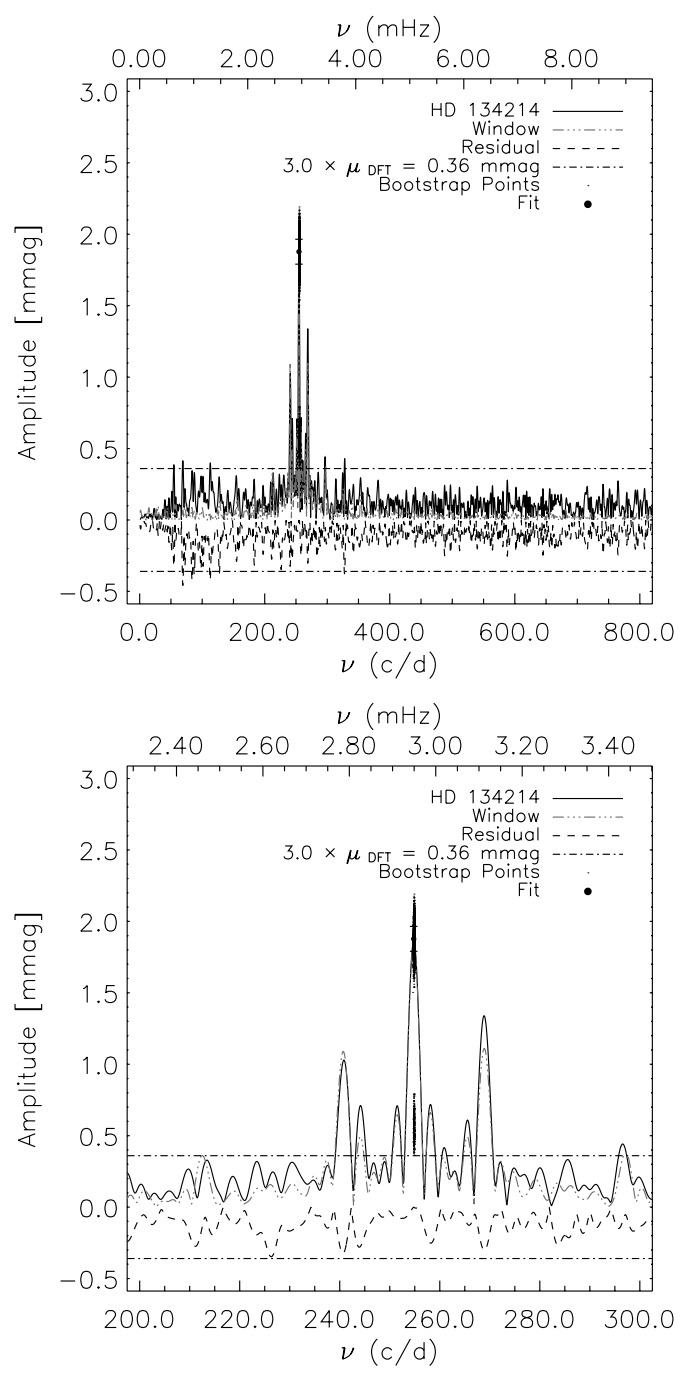

Figure 2: a) (top) The DFT of the HD 134214 data. Shown are the window function, the fit to the data (filled circle) and the bootstrap points (small dots). Also shown is the residual DFT after the fit; inverted for clarity. b) (bottom) The DFT of the data zoomed in on the region of the largest peak. Line styles are the same as in the top plot. 

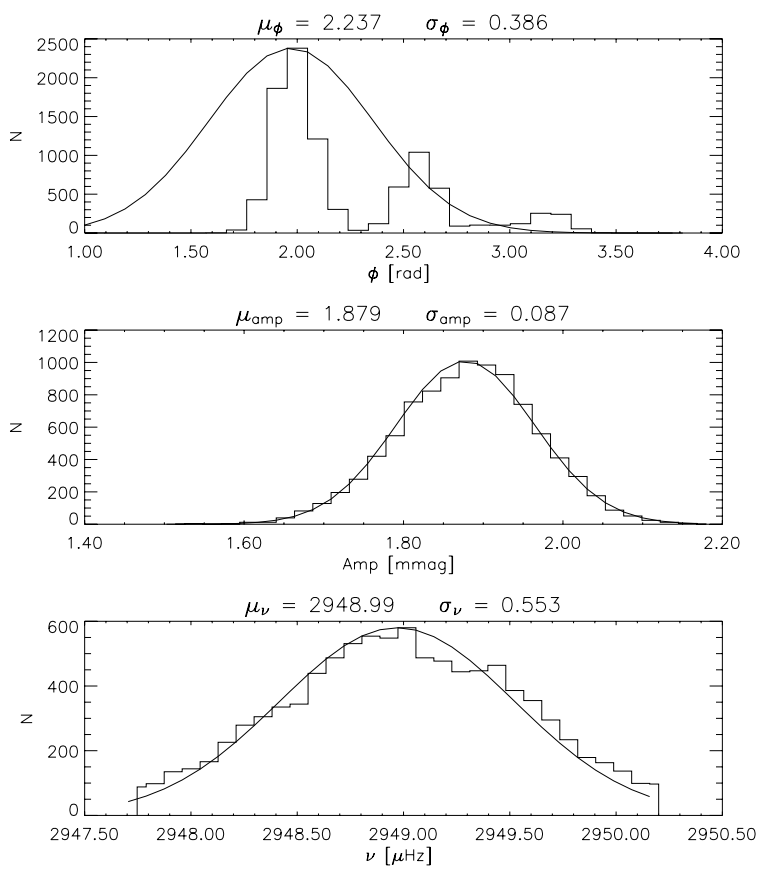

Figure 3: The bootstrap distributions for the fit parameters. Shown from top to bottom are the distributions for the phase $\phi$ in radians, the amplitude in mmag, and the frequency $\nu$ in $\mu \mathrm{Hz}$. The labels above each panel give the mean $\mu$ and the standard deviation $\sigma$ calculated for each distribution. Gaussians with width $\sigma$ and centered on the original fit parameters are plotted over each distribution.

\section{RR Lyrae stars.}

In short, the bootstrap is a technique that allows a user to produce a distribution for each calculated parameter by constructing a large number of light curves from the original data. No assumptions need to be made about noise properties of the data and individual photometric errors are not needed. Each new light curve is assembled by randomly selecting $N$ points from the original light curve (also containing $\mathrm{N}$ points) with the possibility of replacement. In this way, the new synthetic light curves preserve the noise properties of the original data. The fit procedure is repeated for each new light curve, eventually building distributions in each of the fit parameters. We then estimate the $1 \sigma$ error 
bars from the analytic expression for the standard deviation of each distribution under the assumption that they are normally distributed. Each distribution is checked to ensure this assumption is valid.

Figure 3 shows the bootstrap distributions for 10,000 realisations of the fit to our data. The phase is the most uncertain parameter. Amplitude and frequency distributions are normal in shape. Overplotted are Gaussians with the standard deviations derived from the distributions. The Gaussians are centred on the original fit parameters. It should be stressed that bootstrapping only estimates the uncertainties in parameters and does not refine them or assign significances to them.

\section{Discussion}

We determine that HD 134214 is monoperiodic and pulsating with a frequency of $2948.97 \pm 0.55 \mu \mathrm{Hz}$. This is consistent with the earlier photometric observations of this star by Kreidl et al. (1994). Those authors detected frequency changes which they interpreted as cyclic with a timescale of about 1 year. The frequency resolution of our short MOST run is insufficient to rigorously test this assertion. However, we have considered the implications of pulsational frequency variability in HD 134214.

Heller \& Kawaler (1988) have suggested that it may be possible to exploit the frequency variability of roAp stars to determine their evolutionary status. However, the one-year timescale of variability in the case of HD 134214 is too short to be associated with evolution. We have also modelled the amplitude of the frequency change over the main sequence life time of an A-type star. We estimate that a 20 year baseline of observations would show a frequency change on the order of $10^{-4}-10^{-5} \mu \mathrm{Hz}$. This is much smaller than the change of $\sim 0.2 \mu \mathrm{Hz}$ reported by Kreidl et al. (1994). An investigation by Cameron and co-workers has shown, that taking magnetic perturbations to the oscillation frequencies into account does not improve the discrepancy between the observed and calculated frequency changes.

Recent results by Kurtz et al. (2006) suggest that HD 134214 is oscillating spectroscopically in up to 6 modes. Our observations can rule out additional photometric oscillations at a $2 \sigma$ detection level of $0.36 \mathrm{mmag}$. Our estimated DFT noise level of $\sim 0.12$ mmag for 10 hours of observations with MOST is comparable to that obtained by Kreidl et al. (1994) based on about 56 hours of photometry from four observatories during about 4 months. The excitation and selection of pulsation modes in roAp stars is an open question. In the case of HD 134214, there are several avenues to be explored with respect to the additional modes seen spectroscopically: (1) Is it a case of radial velocities of certain elements and ionisation stages being more sensitive to degrees of 
higher $\ell$ ? (2) If the sensitivity of the photometry can be improved sufficiently, will these modes also appear? (3) Is the broadband photometry just averaging over too wide an extent of the pulsating atmosphere? or (4) Might there be new physics in the upper atmospheres of (some) roAp stars to account for the differences observed?

We estimate that even observations over only a few days with MOST would (in addition to improving our frequency resolution enough to investigate cyclic variability) reduce our noise levels by approximately half. Even this modest improvement in noise would be valuable as a test of the Kurtz et al. (2006) theory that photometric observations are not sensitive to the new type of upper atmosphere pulsational variability they report.

Acknowledgments. JMM, DBG, AFJM, SR, and GAHW are supported by funding from the Natural Sciences and Engineering Research Council (NSERC) Canada. RK is funded by the Canadian Space Agency. WWW received financial support from the Austrian Science Promotion Agency (FFG - MOST) and the Austrian Science Funds (FWF - P17580).

\section{References}

Audard, N., et al. 1998, A\&A , 335, 954

Clement, C.M., Jankulak, M., \& Simon, N. R. 1992, ApJ, 395, 192

Heller, C.H., \& Kawaler, S.D. 1988, ApJ, 329, L43

Kreidl, T.J., et al. 1994, MNRAS , 270, 115

Kurtz, D.W. 1982, MNRAS , 200, 807

Kurtz, D.W. 1995, ASP Conf. Ser. 76: GONG 1994. Helio- and Astro-Seismology from the Earth and Space, 76, 60

Kurtz, D.W., \& Martinez, P. 2000, Baltic Astronomy, 9, 253

Kurtz, D.W., Elkin, V.G., \& Mathys, G. 2006, MNRAS , 370, 1274

Kuschnig, R., et al. 1997,A\&A, 328, 544

Lenz, P., \& Breger, M. 2005, CoAst, 146, 53

Matthews, J.M., et al. 2004, Nature, 430, 51

Montgomery, M.H., \& O'Donoghue, D. 1999, DSSN, 13

Rowe, J.F., et al. 2006a, CoAST, 148, 34

Rowe, J.F., et al. 2006b, ApJ , 646, 1241

Saio, H., et al. 2006, ApJ, in press

Shavrina, A., et al. 2004, IAU Symposium, 224, 711

Sperl, M. 1998, CoAst, 111, 1

Walker, G., et al. 2003, PASP, 115, 1023

Walker, G., et al. 2005, ApJ, 635, L77

Wall, J.V., \& Jenkins, C.R. 2003, Practical Statistics for

Astronomers, Cambridge, Cambridge University Press 